\title{
The mediating role of psychological symptoms on falls risk among older adults with osteoarthritis
}

This article was published in the following Dove Press journal:

Clinical Interventions in Aging

\author{
Sumaiyah Mat ${ }^{1}$ \\ Chin Teck Ng ${ }^{1-3}$ \\ Farhana Fadzil ${ }^{4}$ \\ Faizatul Izza Rozalli ${ }^{4}$ \\ Maw Pin Tan ${ }^{1,5}$
}

'Ageing and Age-Associated Disorders Research Group, University of Malaya, Kuala Lumpur, Malaysia; ${ }^{2}$ Department of Rheumatology and Immunology, Singapore General Hospital, ${ }^{3}$ DukeNUS Medical School, National University Singapore, Singapore; ${ }^{4}$ Department of Radiology, Faculty of Medicine, University of Malaya, Kuala Lumpur, Malaysia; ${ }^{5}$ Geriatric Division, Department of Medicine, Faculty of Medicine, University of Malaya, Kuala Lumpur, Malaysia
Correspondence: Maw Pin Tan Geriatric Division, Department of Medicine, Faculty of Medicine, Jalan University, 50603 Kuala Lumpur, Malaysia

Tel +60379493200

Email mptan@ummc.edu.my

\begin{abstract}
The purpose of this study was to investigate the role of fear of falling (FoF) and psychological symptoms in explaining the relationship between osteoarthritis (OA) symptom severity and falls. Individuals aged $\geq 65$ years with $\geq 2$ falls or $\geq 1$ injurious fall over the past 12 months were included in the falls group, while volunteers aged $\geq 65$ years with no history of falls over 12 months were recruited as controls. The presence of lower extremity OA was determined radiologically and clinically. Severity of symptoms was assessed using the Western Ontario and McMaster Universities Arthritis Index (WOMAC) questionnaire. FoF and psychological status were measured with the shortened version of the Falls Efficacy ScaleInternational and the 21-item Depression, Anxiety and Stress Scale (DASS-21), respectively. Of 389 (229 fallers, 160 non-fallers) potential participants, mean (SD) age: 73.74 (6.60) years, 141 had clinical OA and 171 had radiological OA. Fallers with both radiological OA and clinical OA had significantly higher FoF and DASS-21 scores than non-fallers. FoF was significantly positively correlated with symptom severity in fallers and non-fallers with radiological and clinical OA. Depression, anxiety, and stress scores were only significantly correlated with symptom severity among fallers but not non-fallers in both clinical and radiological OA. The relationship between mild symptoms and reduced risk of falls compared to no symptoms in those with radiological OA was attenuated by increased anxiety. The increased falls risk associated with severe symptoms compared to mild symptoms in clinical OA was attenuated by FoF. FoF may, therefore, be a potentially modifiable risk factor for OA-associated falls which could be considered in future intervention studies.
\end{abstract}

Keywords: falls, fear of falling, osteoarthritis, psychological status, aged

\section{Introduction}

Osteoarthritis (OA) is the most prevalent chronic degenerative joint disease world-wide. ${ }^{1}$ Aging is a major risk factor for OA with one in three individuals aged $\geq 75$ years reported to have severe OA changes on their knee radiographs. ${ }^{2}$ Its prevalence, alongside that of many chronic medical conditions, is expected to rise with the rapidly aging global population.

$\mathrm{OA}$ is associated with deficits in balance control. ${ }^{3}$ Among individuals with severe $\mathrm{OA}, 50 \%$ have reported at least one fall in the preceding year. ${ }^{2}$ However, one in three older adults aged $\geq 65$ years also experience at least one fall annually regardless of OA status. ${ }^{4,5}$ Existing published evidence on the relationship between OA and falls have used a variety of different methods for the identification of their OA subjects, including presence of knee pain, self-reported physical diagnosed OA, clinical OA based on agreed criteria, and radiological evidence of OA. These studies also often reported the relationship between $\mathrm{OA}$ and balance measures or falls risk rather than actual fall events. ${ }^{6-9}$ Furthermore, a recent study revealed a biphasic relationship 
between $\mathrm{OA}$ and falls with older individuals with radiological evidence of OA with mild OA symptoms less likely to experience falls compared to those with radiological OA with no symptoms, while individuals with clinical OA and severe symptoms are more likely to experience falls than those with mild symptoms. ${ }^{10}$

Both falls and OA adversely affect the older individuals' psychological well-being and quality of life (QoL). ${ }^{11,12}$ Strong negative associations were observed between depressive mood and fear of falling (FoF) with QoL in older fallers, ${ }^{13}$ while increased pain and functional limitations were found to decrease QoL in patients with OA. ${ }^{11}$

Psychological issues among fallers with OA have not been examined previously. We therefore conducted a casecontrol study comparing older fallers and non-fallers with knee OA, determined using both radiological and clinical criteria, to identify potential differences in FoF and psychological symptoms among fallers and non-fallers with OA.

\section{Methods}

\section{Study population}

This was a cross-sectional case-control study. Subjects aged $\geq 65$ years with either a history of two falls or one injurious fall over the past 12 months were recruited from the Departments of Emergency Medicine, Primary Care, and Geriatric Medicine at a teaching hospital in greater Kuala Lumpur. Control participants with no falls (non-fallers) over the previous 12 months were recruited through media and word-of-mouth advertising. Participants were excluded if they had clinically diagnosed dementia (ICD-10 definition), severe physical disabilities, or major psychiatric illnesses. Demographic details were obtained from all participants. This study was approved by the University of Malaya Medical Centre Medical Ethics Committee (925.4) and complied with the Helsinki Declaration of 1975, revised in 1983. Written informed consent was obtained from all study participants.

\section{Diagnosis of OA}

All participants were assessed for the presence of knee OA using two different definitions of OA, radiological OA and clinical diagnosis of OA, based on predefined criteria. The influence of OA according to each definition on falls was evaluated separately.

\section{Radiographic diagnosis of OA}

Standardized knee radiographs were obtained in the standing antero-posterior view in all consenting participants.
These images were assessed by a radiologist blinded of clinical data. The severity of OA was determined by the Kellgren-Lawrence (KL) grading scale, ${ }^{14}$ with a score of 0 indicating no knee $\mathrm{OA}$ and the maximal score of 4 indicating severe OA. Subjects with KL grades of 2-4 were considered subjects with radiological OA. Participants without radiological OA were excluded in subsequent analysis involving those with radiological OA only.

\section{Clinical diagnosis of $O A$}

Clinical diagnosis of OA was made by a rheumatologist, blinded from clinical data, based on the American College of Rheumatology criteria. Clinical diagnosis of knee OA required the presence of knee arthralgia, plus any 3 of the following: 1) age $>50$ years, 2) morning stiffness, 3 ) crepitus on active motion in at least one side, 4) bony enlargement in at least one side, and 5) no palpable warmth of synovia in both knees. ${ }^{15}$ Participants without clinical OA were excluded from analyses involving those with clinical OA only.

\section{Pain, stiffness, and functional ability}

Pain, stiffness, and functional ability were assessed by the Western Ontario and McMaster Universities Arthritis Index (WOMAC) questionnaire visual analog scale version, in English, Malay, Chinese, or Tamil. The scores for each item were obtained by measuring the distance along the line from the marker at the far left of each analog scale to the point at which the patient's mark intersects the horizontal line on the analog scale. The score of each subscale as well as the grand total score was calculated. The maximum total length or score for pain, stiffness, and physical function subscales were $500 \mathrm{~mm}, 200 \mathrm{~mm}$, and 1,700 mm, respectively. ${ }^{16}$ Individuals with no pain, no stiffness, and no impairment in functional ability were those with a score of zero for pain, stiffness, and physical function. The sum of the individual component scores for pain, stiffness, and physical function was calculated to obtain the total WOMAC score. Using 25 th, 50th, and 75th percentile values as ordinal cutoffs, the non-normally distributed total WOMAC score was further categorized into 4 groups: no symptoms ( $0 \mathrm{~mm})$, mild symptoms (1-200 mm), moderate symptoms (201-465 mm), and severe symptoms ( $\geq 466 \mathrm{~mm})$.

\section{Falls efficacy and psychological status FoF}

The shortened version of the Falls Efficacy Scale-International (short FES-I) was used. The short FES-I consists of seven items on a 4-point Likert scale, with 1 indicating no concern 
and 4 indicating severe concern. The minimum and maximum scores for the short FES-I are therefore 7 and 28, respectively. ${ }^{17}$ To allow for adjustment for potential confounders, cutoffs were developed using the median values; subjects with a score of $\geq 11$ were considered to have greater FoF.

\section{Depression, anxiety, and stress}

Depression, anxiety, and stress were measured with the 21-item Depression, Anxiety, and Stress Scale (DASS-21). This is a self-reported measure in which subjects rate the frequency and severity of the negative emotions of depression, anxiety, and stress over the previous week. Frequency or severity ratings are made on a series of 4-point scales, with 0 indicating "did not apply to me at all" and 3 indicating "applied to me very much, or most of the time." 18 The scores were calculated individually for the three components: depression, anxiety, and stress. Because the DASS-21 is the short version of DASS (which has 42 items), the final score for each item were multiplied by $2 .{ }^{19}$ The total score for each components was dichotomized using median values as the cutoffs, ${ }^{20}$ depression $\geq 4$, anxiety $\geq 4$, and stress $\geq 8$, respectively.

\section{Statistical analysis}

The SPSS 20.0 (IBM Corporation, Armonk, NY, USA) statistical package was used for statistical analysis. Normality was assessed using the Kolmogorov-Smirnov test. Mean values with $\mathrm{SD}$ or median with interquartile range were expressed for normally and non-normally distributed continuous data accordingly. The independent $t$-test and Mann-Whitney $U$ test were employed for parametric and non-parametric data, respectively. Categorical variables were expressed as frequencies with percentages in parentheses and compared with the chi-squared test. Spearman's rank correlation was used to evaluate the correlation between WOMAC subscales with FoF and DASS-21 domain scores. Using cutoffs described earlier, continuous data were grouped into dichotomous variables. Subsequently, logistic regression analysis using fall as a dependent variable was performed to determine potentially mediating effects of FoF and psychological status on the relationship between OA severity and falls. Dummy variables were created for comparisons between multiple categories, with the lowest category (no arthritis or mild arthritis) as the reference category. The exponential value of the parameter estimate, $B$, for each variable, which would represent the adjusted odds ratio (OR) as a measure of the strength of association was presented along with its $95 \% \mathrm{CI}$.

\section{Results Demographics}

A total of 389 subjects, 229 fallers and 160 non-fallers, with a mean age of $72.2 \pm 6.1$ years, were evaluated and screened for the presence of OA. One hundred and forty-one $(36.2 \%)$ individuals had clinically diagnosed OA based on the ACR criteria. Knee X-rays were available for 205 participants, and $171(83 \%)$ had radiological evidence of OA. Characteristics of fallers and non-fallers with radiological and clinical OA are summarized in Table 1. Fallers were significantly older than non-fallers in both the groups. There were ethnic differences between fallers and non-fallers with clinical OA. A greater proportion of fallers compared to non-fallers had severe OA symptoms in clinical OA. A higher percentage of non-fallers were seen with "mild" OA symptoms in radiological OA, compared to asymptomatic OA (Table 1).

\section{FoF and psychological status among fallers and non-fallers with different types of OA}

In Table 2, fallers with either radiological or clinical OA had significantly greater FoF and reported significantly more severe depressive symptoms than non-fallers. Due to the non-normally distributed data, the individual domain scores were then dichotomized using median cutoffs to allow for adjustment for potential confounders using logistic regression analyses. After adjustment for the potential confounders of age, gender, ethnicity, and comorbidities, the association between greater FoF and falls remained significant in both the types of OA. However, the association between depression and falls in radiological OA group was no longer statistically significant after the same adjustment (Table 2).

\section{FoF and psychological domains correlated with OA symptom severity in fallers and non-fallers}

Table 3 documents the correlation coefficients (Spearman's rho) between FoF, psychological status, and grand total of WOMAC score in fallers and non-fallers who had OA, diagnosed radiologically or clinically. Among fallers with radiological OA or clinical OA, symptom severity was significantly and positively correlated with $\mathrm{FoF}$ and all three psychological domains $(p<0.01)$. In contrast, among the non-fallers, symptom severity was only significantly correlated with FoF regardless of the method of OA diagnosis. Symptom severity correlated with depression only in those with radiological OA (Table 3). 
Table I Characteristics of individuals with radiological OA and clinical OA according to fall status

\begin{tabular}{|c|c|c|c|c|c|c|}
\hline & \multicolumn{3}{|c|}{ Radiological OA (n=|7I) } & \multicolumn{3}{|c|}{ Clinical OA $(n=141)$} \\
\hline & $\begin{array}{l}\text { Fallers } \\
(n=106)\end{array}$ & $\begin{array}{l}\text { Non-fallers } \\
(n=65)\end{array}$ & $p$-value & $\begin{array}{l}\text { Fallers } \\
(n=8 I)\end{array}$ & $\begin{array}{l}\text { Non-fallers } \\
(n=60)\end{array}$ & $p$-value \\
\hline Age (years), mean (SD) & $75.28(6.92)$ & 71.31 (4.65) & $<0.001$ & $75.93(6.85)$ & $71.98(5.54)$ & $<\mathbf{0 . 0 0 I}$ \\
\hline Female sex, n (\%) & $75(70.8)$ & $50(76.9)$ & 0.377 & $58(71.6)$ & $47(78.3)$ & 0.365 \\
\hline BMI $\left(\mathrm{kg} / \mathrm{m}^{2}\right)$, mean $(\mathrm{SD})$ & $24.73(4.22)$ & $24.42(3.34)$ & 0.623 & $25.05(4.3 \mathrm{I})$ & $25.42(3.83)$ & 0.601 \\
\hline \multicolumn{7}{|l|}{ Ethnicity*, n (\%) } \\
\hline Malay & $14(13.2)$ & $12(18.5)$ & Reference & II (I3.6) & $14(23.3)$ & Reference \\
\hline Chinese & $71(67.0)$ & $46(70.8)$ & 0.521 & $52(64.2)$ & $40(66.7)$ & 0.268 \\
\hline Indian & $17(16.0)$ & $4(6.2)$ & 0.058 & $16(19.8)$ & $3(5.0)$ & 0.010 \\
\hline Others & $4(3.8)$ & $3(4.6)$ & 0.876 & $2(2.5)$ & $3(5.0)$ & 0.869 \\
\hline \multicolumn{7}{|l|}{ Comorbidities, n (\%) } \\
\hline Heart diseases & $9(8.5)$ & $3(4.6)$ & 0.336 & $7(8.6)$ & $4(6.8)$ & 0.769 \\
\hline Hypertension & $57(53.8)$ & $34(52.3)$ & 0.852 & $52(64.2)$ & $31(52.5)$ & 0.166 \\
\hline Diabetes mellitus & $32(30.2)$ & $13(20.0)$ & 0.142 & $26(32.1)$ & $13(22.0)$ & 0.190 \\
\hline Stroke & $7(6.6)$ & $3(4.6)$ & 0.591 & $5(6.2)$ & $2(3.4)$ & 0.699 \\
\hline Atrial fibrillation & $2(1.9)$ & I (I.5) & 1.000 & $2(2.5)$ & I ( 1.7$)$ & 1.000 \\
\hline Visual impairment & $26(24.5)$ & II (16.9) & 0.241 & $18(22.2)$ & $12(20.3)$ & 0.789 \\
\hline \multicolumn{7}{|c|}{ WOMAC grand total score*, n (\%) } \\
\hline No symptoms & $27(25.5)$ & $16(24.6)$ & Reference & 0 & 0 & NA \\
\hline Mild & $19(17.9)$ & $28(43.1)$ & 0.036 & $18(22.2)$ & $25(4 \mid .7)$ & Reference \\
\hline Moderate & $25(23.6)$ & $13(20.0)$ & 0.779 & $21(25.9)$ & $22(36.7)$ & 0.516 \\
\hline Severe & $35(33.0)$ & $8(12.3)$ & 0.058 & $42(51.9)$ & $13(21.7)$ & 0.001 \\
\hline
\end{tabular}

Notes: *Logistic regression with dummy variables. Categorized using percentile cutoffs from total WOMAC score. "No symptoms": 0 mm. "Mild": I-200 mm. "Moderate": 20I-465 mm. "Severe": $\geq 466 \mathrm{~mm}$. Mean age and BMI were compared using Student's independent $t$-test. The bold font indicates statistical significance.

Abbreviations: BMI, body mass index; OA, osteoarthritis; IQR, interquartile range; FES-I, Falls Efficacy Scale-International; DASS-2I, 2I-item Depression, Anxiety, and Stress Scale; WOMAC, Western Ontario and McMaster Universities questionnaire; NA, not applicable.

\section{Multivariate logistic regression evaluating the relationship between OA severity and falls}

Table 4 explores the mediating roles of FoF and psychological domains on the association between symptom severity and falls according to OA diagnosis. Models 1-5 assessed the relationship between symptom severity and falls in those with radiological OA, while models $6-10$ explored the relationship between symptom severity and falls in those with clinical OA.

\section{Symptom severity and falls in radiological OA}

Comparisons were made with falls as the dependent variable and categorical severity of $\mathrm{OA}$ as independent variables using no OA symptoms as the reference group. In Model 1, following adjustments for the potential confounders of age, gender, ethnicity, and comorbidities, mild OA symptoms in radiological OA was associated with reduced risk of falls (OR $=0.38,95 \% \mathrm{CI}=0.15$ to -0.98$)$ compared to those with radiological OA and no symptoms. No significant difference in falls risk was observed in those with moderate OA symptoms or severe OA symptoms compared to those with no symptoms in this model. In Model 2, the protective relationship between mild OA symptoms remained after adjustments were made for short FES-I scores ( $\mathrm{OR}=0.33$, $95 \% \mathrm{CI}=0.12$ to 0.87 ). This relationship also remained statistically significant after adjustment for DASS depression (Model 3: OR $=0.38,95 \% \mathrm{CI}=0.15$ to 0.97 ) and DASS stress scores (Model 4: $\mathrm{OR}=0.39,95 \% \mathrm{CI}=0.15$ to 0.97 ). However, adjustment for DASS anxiety scores led to attenuation of the protective effect of mild OA symptoms against falls compared to no OA symptoms in those with radiological OA (Model 4: OR $=0.40,95 \% \mathrm{CI}=0.16$ to 1.01 ).

\section{Symptom severity and falls in clinical OA}

Comparisons were made using falls as the dependent variable and OA symptom severity category. As the presence of symptom is requisite for the diagnosis of clinical OA, OA symptom severity as determined by the WOMAC scores was categorized only according to the mild, moderate, and severe categories. Model 6 suggests that individuals with clinical OA and severe OA symptoms were significantly more likely to experience falls compared to those with mild OA symptoms ( $\mathrm{OR}=3.92,95 \% \mathrm{CI}=1.51$ to 10.20$)$. This relationship was attenuated after adjustment for short FES-I scores ( $\mathrm{OR}=2.56,95 \% \mathrm{CI}=0.90$ to 7.28 ). However, DASS depression, anxiety, and stress scores did 


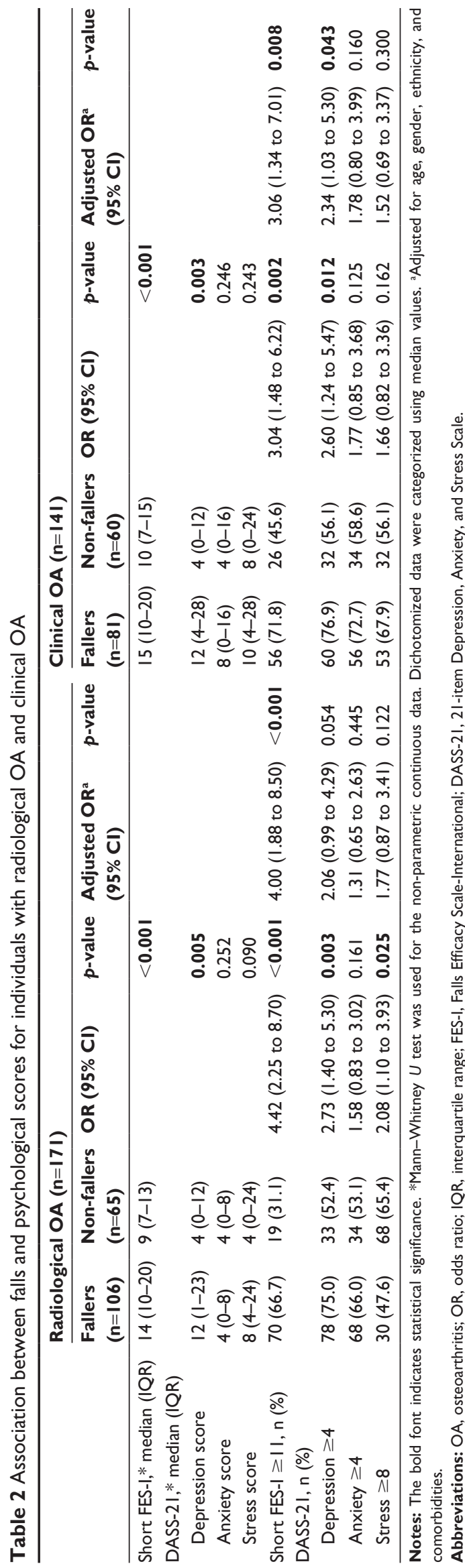

Table 3 Correlation between short FES-I and DASS-2I scores and total WOMAC scores among fallers and non-fallers, with radiological and clinical $O A$

\begin{tabular}{llllll}
\hline \multirow{2}{*}{$\begin{array}{l}\text { Total WOMAC } \\
\text { score }\end{array}$} & \multicolumn{2}{l}{ Radiological OA } & & \multicolumn{2}{c}{ Clinical OA } \\
\cline { 2 - 3 } \cline { 5 - 6 } \cline { 5 - 6 } & Fallers & Non-fallers & & Fallers & Non-fallers \\
\hline Short FES-I & $0.375^{* *}$ & $0.5 I 5^{* *}$ & & $0.453^{* *}$ & $0.397^{* *}$ \\
DASS-2I & & & & & \\
$\quad$ Depression & $0.439^{* *}$ & $0.258^{*}$ & & $0.469^{* *}$ & 0.177 \\
$\quad$ Anxiety & $0.287^{* *}$ & 0.069 & & $0.24 I^{* *}$ & 0.147 \\
$\quad$ Stress & $0.256^{* *}$ & 0.149 & & $0.358^{* *}$ & 0.222 \\
\hline
\end{tabular}

Notes: $* * p<0.01 ; * p<0.05$.

Abbreviations: WOMAC, Western Ontario and McMaster Universities questionnaire; FES-I, Falls Efficacy Scale-International; DASS-2I, 2 I-item Depression, Anxiety, and Stress Scale; OA, osteoarthritis.

not influence the relationship between severe OA symptoms in those with clinical OA and falls (Models 8-10). Those with moderate OA symptoms did not display any difference in falls risk compared to those with mild OA symptoms in all models (Table 4).

\section{Discussion}

Among individuals with $\mathrm{OA}$, fallers were significantly more likely to experience FoF and depression, regardless of whether the presence of OA was determined using radiological imaging or clinical assessment. When the relationship between severity of OA symptoms and psychological status were assessed independently among fallers and non-fallers, however, there was a significant correlation between severity of depression, anxiety, and stress with OA severity scores in fallers regardless of diagnostic criteria, but not non-fallers. Further multivariate analyses revealed that the increased risk of falls associated with severe clinical OA was mediated by FoF while the protective effect of mild symptoms in those with radiological OA was in fact mediated by symptoms of anxiety. Our findings, therefore, highlight a potential role of psychological status and FoF in determining the risk of falls in those older individuals with OA.

Our study evaluated the psychological factors that influence falls risk in individuals with OA using two established methods of determining the presence of OA. Published studies have previously established the relationship between psychological status, including depression and FoF and increased risk of falls among general older populations. ${ }^{21}$ The relationship between falls and clinically significant depressive symptoms was also identified in subjects with self-reported OA in the Health and Retirement study. ${ }^{22}$ In a separate study involving those with clinical OA, a high level of anxiety was associated with poorer self-reported physical function but not gait and balance impairment quantified by 
Table 4 The effects of fear of falling and psychological status in the relationship between symptom severity and falls

\begin{tabular}{|c|c|c|c|c|c|}
\hline & \multicolumn{5}{|l|}{ Falls OR $(95 \% \mathrm{Cl})$} \\
\hline & Model Ia & Model $2^{b}$ & Model 3c & Model $4^{d}$ & Model $5^{\mathbf{e}}$ \\
\hline \multicolumn{6}{|c|}{ Radiological OA $(n=|7|)$} \\
\hline \multicolumn{6}{|c|}{ No symptoms (ref) } \\
\hline Mild & $0.386(0.15$ to 0.98$)$ & $0.33(0.12$ to 0.87$)$ & $0.38(0.15$ to 0.97$)$ & $0.40(0.16$ to 1.01$)$ & $0.39(0.15$ to 0.97$)$ \\
\hline Moderate & $0.99(0.36$ to 2.70$)$ & $0.59(0.19$ to 1.81$)$ & $0.95(0.35$ to 2.62$)$ & 1.02 (0.36 to 2.89$)$ & $0.97(0.35$ to 2.66$)$ \\
\hline \multirow[t]{2}{*}{ Severe } & 2.45 (0.84 to 7.16$)$ & $\mathrm{I} .14(0.37$ to 3.84$)$ & $2.63(0.85$ to 8.13$)$ & 2.95 (0.94 to 9.22$)$ & 2.74 (0.90 to 8.39$)$ \\
\hline & Model 6a & Model $7^{b}$ & Model 8c & Model 9d & Model I0e \\
\hline \multicolumn{6}{|c|}{ Clinical OA $(n=|4|)$} \\
\hline No symptoms & NA & NA & NA & NA & NA \\
\hline \multicolumn{6}{|l|}{ Mild (ref) } \\
\hline Moderate & $1.29(0.5 \mathrm{I}$ to 3.27$)$ & 0.93 (0.34 to 2.52$)$ & 1.35 (0.53 to 3.47$)$ & I. .46 (0.56 to 3.79$)$ & $\mathrm{I} .36(0.53$ to $3.5 \mathrm{I})$ \\
\hline Severe & $3.92(1.5 \mid$ to $\mid 0.20)$ & $2.56(0.90$ to 7.28$)$ & 3.7 ( $(1.37$ to 10.07$)$ & 4.33 (1.56 to 12.00$)$ & $4.06(1.49$ to $I 1.07)$ \\
\hline
\end{tabular}

Notes: Bold font indicates statistical significance. No symptoms $=0 \mathrm{~mm}$, mild symptoms $=\mathrm{I}-200 \mathrm{~mm}$, moderate symptoms $=20 \mathrm{I}-465 \mathrm{~mm}$, and severe symptoms $\geq 466 \mathrm{~mm}$. ${ }^{a}$ Adjusted for age, gender, ethnicity, and comorbidities. ${ }^{b}$ Adjusted for age, gender, ethnicity, comorbidities, and fear of falling (short FES-I). 'Adjusted for age, gender, ethnicity,

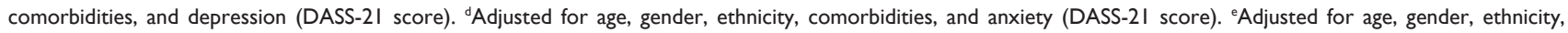
comorbidities, and stress (DASS-2I score).

Abbreviations: NA, not applicable; OA, osteoarthritis; OR, odds ratio.

physical performance measure of function. ${ }^{23}$ As highlighted earlier, these previous studies had used varying definitions of OA and may not necessarily employ actual fall events as their primary outcome. Our study has therefore emphasized that psychological factors do play a role in falls risk regardless of OA definitions. In addition, our study had compared actual fallers with non-fallers rather than employing surrogate measures such as balance performance, as in previous studies.

The etiology of psychological symptoms in OA and the increased psychological burden in fallers with OA has not been well elucidated. Pain and joint stiffness associated with $\mathrm{OA}$ are chronic, though often branded less severe than other inflammatory joint conditions. The long-term effects of joint pain and stiffness could have a direct consequence on mood. ${ }^{24}$ In addition, a previous study has demonstrated a significant association between FoF and pain. ${ }^{25}$ The presence of OA, however, also influences joint stability, which occurs as a result of ligamentaous laxity from deconditioning occurring as a consequence of activity avoidance due to joint symptoms. Knee buckling, which occurs as a result of joint instability, is in turn, associated with FoF. ${ }^{26}$ Furthermore, joint pain and stiffness, instability, FoF, and reduced physical activity all lead to reduced functional ability, which will also adversely affect psychological well-being. The increased risk of $\mathrm{FoF}$ and depression among fallers with OA compared to non-fallers may imply that fallers were either more likely to experience the adverse psychological consequences of OA or that fallers with OA experience additional psychological burden over non-fallers with OA. However, when further within group analyses were employed, while the dose-response relationship is observed between FoF and OA symptom severity in both fallers and non-fallers with OA, the dose-response relationship between depression, stress, and anxiety and OA symptom severity only among fallers. The cross-sectional design of our study does not allow for the determination of the temporal relationships between FoF and depression with falls. ${ }^{24}$

We further explored the effects of FoF, depression, anxiety, and stress on the differential relationship of severity of OA symptoms with falls using multivariate logistic regression. The reduced risk of falls observed among those with radiological OA and mild symptoms had previously been reported. ${ }^{10}$ Our mediation analysis, however, suggests that symptoms of anxiety may account for the reduced falls risk in those with radiological OA and mild symptoms over those with radiological OA and no symptoms. This suggests that those with increased anxiety may exhibit increased vigilance or avoidance of challenging activities which then reduces their risk of falling. ${ }^{27,28}$ The exposure of these individuals to extrinsic risk factors for falls such as physical environment, assistive devices, or footwear may also be reduce. ${ }^{21}$

Conversely, while individuals with clinical OA and severe symptoms were significantly more likely to experience falls than those with mild symptoms, this relationship was not influenced by psychological status. Our findings were consistent with that of Quach and Burr who also found no interaction between clinically significant depressive symptoms with falls in their subjects with OA. ${ }^{22} \mathrm{FoF}$, on the other hand, seemed to account for the excess falls risk associated with severe OA compared to mild OA among individuals with 
clinical OA. Our cross-sectional findings, as before, does not allow us to determine any causal relationship between FoF and falls in individuals with clinical OA, but does, however, highlight FoF as a potentially modifiable risk factor for falls among those with severe clinical OA. A recent systematic review suggested that exercise interventions may reduce FoF in community-dwelling general older populations. ${ }^{29}$ Now, the use of actual psychological interventions in the management of FoF is also being evaluated. ${ }^{30}$ The determination of the value of exercise and psychological interventions on FoF and falls may therefore be worthwhile in individuals with clinical OA.

As mentioned earlier, the cross-sectional design of our study precludes the establishment of temporality or causation. Fallers in our study also consisted of those with recurrent or injury falls, who will be considered high-risk fallers. The risk of injury and healthcare burden are particularly high among this group of fallers, which may limit the applicability of our findings to those who sustain one accidental fall with no injury, but may suffer adverse psychosocial consequence as a result. In addition, the presence of falls in the previous 12 months was determined through self-report, which may be subject to recall bias. ${ }^{31} \mathrm{~A}$ larger, more robust, prospective study to help establish the temporal relationship between FoF and other psychological symptoms and falls in individuals with OA should now be conducted to aid further understanding of the complex relationships between psychological status, OA, and falls. ${ }^{32,33}$

\section{Conclusion}

Older fallers with radiological and clinical OA have an increased FoF and depressive symptoms compared to nonfallers. A dose-response relationship exists between FoF, depression, anxiety, and stress with OA symptom severity among fallers with OA. Further exploratory analyses suggest mediating effects of anxiety on the protective effect of mild symptoms among those with radiological OA and FoF on the increased risk of falls among those with severe clinical OA. Our findings highlight the important role of psychological status in falls among older individuals with OA, which will help determine the direction of future studies on strategies to reduce the burden on falls in those with OA.

\section{Acknowledgments}

We thank Dr Karen Morgan for her valuable input during the preparation of this manuscript. This study has been funded by a University of Malaya Grand Challenge Research Fund
(GC002-14HTM) and a University of Malaya Postgraduate Research Fund (PPP) grant (PG013-2014B).

\section{Disclosure}

The study sponsors had no role in the collection, analysis and interpretation of data; in the writing of the manuscript; or in the decision to submit the manuscript for publication. The authors report no conflicts of interest in this work.

\section{References}

1. Zhang Y, Jordan JM. Epidemiology of osteoarthritis. Clin Geriatr Med. 2010;26:355-369.

2. Arden N, Nevitt MC. Osteoarthritis: epidemiology. Best Pract Res Clin Rheumatol. 2006;20:3-25.

3. Kim HS, Yun DH, Yoo SD, et al. Balance control and knee osteoarthritis severity. Ann Rehabil Med. 2011;35:701-709.

4. Murray CJ, Barber RM, Foreman KJ, et al. Global, regional, and national disability-adjusted life years (DALYs) for 306 diseases and injuries and healthy life expectancy (HALE) for 188 countries, 1990-2013: quantifying the epidemiological transition. Lancet (London, England). 2015;386:2145-2191.

5. Tromp AM, Pluijm SM, Smit JH, Deeg DJ, Bouter LM, Lips P. Fall-risk screening test: a prospective study on predictors for falls in communitydwelling elderly. J Clin Epidemiol. 2001;54:837-844.

6. Ng CT, Tan MP. Osteoarthritis and falls in the older person. Age Ageing. 2013;42:561-566.

7. Levinger P, Menz HB, Wee E, Feller JA, Bartlett JR, Bergman NR. Physiological risk factors for falls in people with knee osteoarthritis before and early after knee replacement surgery. Knee Surg Sports Traumatol Arthrosc. 2011;19:1082-1089.

8. Foley SJ, Lord SR, Srikanth V, Cooley H, Jones G. Falls risk is associated with pain and dysfunction but not radiographic osteoarthritis in older adults: Tasmanian Older Adult Cohort study. Osteoarthritis Cartilage. 2006; 14:533-539.

9. Khalaj N, Abu Osman NA, Mokhtar AH, Mehdikhani M, Wan Abas WA. Balance and risk of fall in individuals with bilateral mild and moderate knee osteoarthritis. PLoS One. 2014;9:e92270.

10. Mat S, Tan PJ, Ng CT, et al. Mild joint symptoms are associated with lower risk of falls than asymptomatic individuals with radiological evidence of osteoarthritis. PLoS One. 2015;10:e141368.

11. Jakobsson U, Hallberg IR. Pain and quality of life among older people with rheumatoid arthritis and/or osteoarthritis: a literature review. J Clin Nurs. 2002;11:430-443.

12. Al-Ahaideb A, Alrushud A, El-Sobkey S, Hafez A. Impact of knee osteoarthritis on the quality of life among Saudi elders: a comparative study. Saudi J Sports Med. 2013;13:10.

13. Thiem R, Klaßßen-Mielke R, Trampisch U, Moschny A, Pientka L, Hinrichs T. Falls and EQ-5D rated quality of life in community-dwelling seniors with concurrent chronic diseases: a cross-sectional study. Health Qual Life Outcomes. 2014;12:2.

14. Kellgren JH, Lawrence JS. Radiological assessment of osteo-arthrosis. Ann Rheum Dis. 1957;16:494-502.

15. Edwards $\mathrm{MH}$, van der Pas S, Denkinger MD, et al. Relationships between physical performance and knee and hip osteoarthritis: findings from the European Project on Osteoarthritis (EPOSA). Age Ageing. 2014;43:806-813.

16. Bellamy N. WOMAC Osteoarthritis Index User Guide. Version X. Queensland, Australia; 2012:79.

17. Kempen GI, Yardley L, van Haastregt JC, et al. The Short FES-I: a shortened version of the falls efficacy scale-international to assess fear of falling. Age Ageing. 2008;37:45-50. 
18. Oei TP, Sawang S, Goh YW, Mukhtar F. Using the Depression Anxiety Stress Scale 21 (DASS-21) across cultures. Int J Psychol. 2013;48: 1018-1029.

19. Lovibond PF, Lovibond SH. The structure of negative emotional states: comparison of the Depression Anxiety Stress Scales (DASS) with the Beck Depression and Anxiety Inventories. Behav Res Ther. 1995;33: 335-343.

20. MacCallum RC, Zhang S, Preacher KJ, Rucker DD. On the practice of dichotomization of quantitative variables. Psychol Methods. 2002; 7:19-40.

21. Tinetti ME, Kumar C. The patient who falls: "It's always a trade-off". JAMA. 2010;303:258-266.

22. Quach LT, Burr JA. Arthritis, depression, and falls among communitydwelling older adults: evidence from the health and retirement study. J Appl Gerontol. 2016 Epub May 1.

23. Scopaz KA, Piva SR, Wisniewski S, Fitzgerald GK. Relationships of fear, anxiety, and depression with physical function in patients with knee osteoarthritis. Arch Phys Med Rehabil. 2009;90:1866-1873.

24. Eggermont LH, Penninx BW, Jones RN, Leveille SG. Depressive symptoms, chronic pain, and falls in older community-dwelling adults: the MOBILIZE Boston Study. J Am Geriatr Soc. 2012;60:230-237.

25. Patel KV, Phelan EA, Leveille SG, et al. High prevalence of falls, fear of falling, and impaired balance in older adults with pain in the United States: findings from the 2011 National Health and Aging Trends Study. J Am Geriatr Soc. 2014;62:1844-1852.

26. Nguyen US, Felson DT, Niu J, et al. The impact of knee instability with and without buckling on balance confidence, fear of falling and physical function: the multicenter osteoarthritis study. Osteoarthritis Cartilage. 2014;22:527-534.
27. Nguyen USD, Zhang Y, VanderWeele TJ, et al. Does physical performance mediate the effect of knee osteoarthritis and risk of indoor and outdoor falls in older men and women? [abstract]. Arthritis Rheum. 2011;63:797.

28. Trivedi MH. The link between depression and physical symptoms. Primary Care Companion J Clin Psychiatry. 2004;6:12-16.

29. Kumar A, Delbaere K, Zijlstra GA, et al. Exercise for reducing fear of falling in older people living in the community: Cochrane systematic review and meta-analysis. Age Ageing. 2016;45:345-352.

30. Parry SW, Bamford C, Deary V, et al. Cognitive-behavioural therapybased intervention to reduce fear of falling in older people: therapy development and randomised controlled trial - the Strategies for Increasing Independence, Confidence and Energy (STRIDE) study. Health Technol Assess. 2016;20:1-206.

31. Cummings SR, Nevitt MC, Kidd S. Forgetting falls. The limited accuracy of recall of falls in the elderly. J Am Geriatr Soc. 1988;36:613-616.

32. Murphy SL, Dubin JA, Gill TM. The development of fear of falling among community-living older women: predisposing factors and subsequent fall events. J Gerontol A Biol Sci Med Sci. 2003;58: M943-M947.

33. Murphy SL, Williams CS, Gill TM. Characteristics associated with fear of falling and activity restriction in community-living older persons. J Am Geriatr Soc. 2002;50:516-520.
Clinical Interventions in Aging

\section{Publish your work in this journal}

Clinical Interventions in Aging is an international, peer-reviewed journal focusing on evidence-based reports on the value or lack thereof of treatments intended to prevent or delay the onset of maladaptive correlates of aging in human beings. This journal is indexed on PubMed Central, MedLine,

\section{Dovepress}

CAS, Scopus and the Elsevier Bibliographic databases. The manuscript management system is completely online and includes a very quick and fair peer-review system, which is all easy to use. Visit http://www.dovepress. com/testimonials.php to read real quotes from published authors. 